\title{
Synesthetic associations and psychosensory symptoms of temporal epilepsy
}

\author{
This article was published in the following Dove Press journal: \\ Neuropsychiatric Disease and Treatment \\ II January 2016 \\ Number of times this article has been viewed
}

\section{Marcel Neckar \\ Petr Bob}

Center for Neuropsychiatric Research of Traumatic Stress, Department of Psychiatry, First Faculty of Medicine, Charles University, Prague, Czech Republic
Correspondence: Petr Bob Center for Neuropsychiatric Research of Traumatic Stress, Department of Psychiatry, First Faculty of Medicine, Charles University, Ke Karlovu I I, I 2000 Prague 2, Czech Republic Email petrbob@netscape.net
Background: Synesthesia manifests as unusual associative connections that may cause intriguing experiences due to various cross-modal connections, for example, a sound may be experienced as color. Several findings indicate that temporal lobe seizures or seizure-like conditions and increased excitability may influence various unusual cross-sensory links and synesthetic experiences.

Methods: In this context, the purpose of this study is to find relationships between word-color associations and psychopathological symptoms related to temporal lobe epilepsy and limbic irritability (Limbic System Checklist [LSCL-33]), symptoms of traumatic stress (Trauma Symptoms Checklist [TSC-40]), and depressive symptoms (Beck Depression Inventory [BDI-II]) in 71 participants (mean age $=25.23$ years) recruited from the general population. The whole sample included two subgroups according to levels of psychosensory and affective symptoms related to temporal epilepsy measured by LSCL-33.

Results: The results in both subgroups indicate specific words correlated with the scores of psychopathological symptoms measured by LSCL-33, BDI-II, and TSC-40. Significant Spearman correlations have been predominantly found in the subgroup of participants with higher levels of LSCL-33.

Conclusion: The results indicate a specific synesthetic-like mechanism in association processes that reflects psychopathological symptoms related to increased temporo-limbic excitability.

Keywords: word associations, colors, stress, synesthesia, temporal lobe epilepsy, limbic irritability

\section{Introduction}

Temporal lobe epilepsy represents the neurological pathological condition, which is also related to a wide spectrum of psychopathological symptoms that manifest as various sensory distortions, hallucinatory experiences, depersonalization, derealization and other psychosensory, and affective symptoms usually reported in patients with temporal lobe epilepsy. ${ }^{1-4}$ Recent research indicates that these symptoms may occur as a continuum of complex partial seizure-like symptoms also in patients with mental disorders and in the normal population. ${ }^{1,4}$ Some data also show that these symptoms may be related to stressful and traumatic experiences that may influence inhibitory functions and neural excitability mainly in the limbic system. ${ }^{1-3}$ Several findings also show that temporal lobe seizures or seizure-like conditions may manifest in various unusual cross-sensory links and unusual associative connections that may cause synesthetic phenomena. ${ }^{5,6}$ Synesthesia is a phenomenon related to various forms of inter-sensory connections, for example, hearing a sound may evoke seeing a color. . $^{, 7,8}$

According to recent findings, synesthesia in its mild forms may have relatively high prevalence in the population, which according to some reported data may be at approximately $30 \%-50 \% .^{7,9}$ Recent studies indicate that the phenomenon of the 
so-called "soft synesthesia" may be related to various forms of associative connections as, for example, grapheme-color synesthesia $^{10-11}$ or sound-color synesthesia where higher sounds evoke images of lighter and brighter colors and vice versa. ${ }^{10,12}$ Several studies documented that lighter colors are more frequently associated with positive emotional meanings (eg, happy, good) and certain color-specific associations were found for red color ("strong" and "angry"), blue ("good"), green ("strong"), purple ("sad"). ${ }^{11,13}$

In this context, the purpose of this study is to assess relationships between mild forms of temporal lobe psychosensory symptoms and word-color synesthetic experience using a novel method of word-color associations. This method reflects an emotional response to words that according to their emotional meaning may be specifically associated with darker or lighter color and quantified on a Likert scale. These findings suggest a hypothesis that colors related to emotions in response to certain specific words could be more associated with the actual presence of psychosensory symptoms related to temporal lobe epilepsy than with other psychopathological symptoms. For the purpose to test the hypothesis we have compared two subgroups of participants selected from the general population. The first subgroup of participants who had higher level of psychosensory and affective symptoms related to temporal epilepsy was compared with a healthy control subgroup of participants who had only minor level of these symptoms.

\section{Participants and methods} Participants

The sample included 71 participants (mean age $=25.23$; $\mathrm{SD}=7.21$, age range: $18-39$ years) selected by advertising from the general population and consisted of 41 women and 30 men predominantly with high school education. The whole sample included two subgroups according to levels of psychosensory and affective symptoms related to temporal epilepsy measured by Limbic System Checklist (LSCL-33). The first subgroup $(\mathrm{N}=31)$ included participants with LSCL-33 score 28 or more and the second subgroup ( $\mathrm{N}=40)$ included participants with LSCL-33 score less than 10. All participants signed informed consent and the study was approved by Charles University ethical committee.

\section{Methods}

Symptoms similar to ictal temporal lobe epilepsy, such as somatic, sensory, behavioral, and memory symptoms linked to temporal lobe epileptiform activity, were assessed by LSCL-33. ${ }^{1}$ LSCL-33 is designed to measure temporolimbic activity in the form of somatic, sensory, behavioral, and memory symptoms known to be associated with the phenomena of ictal temporal lobe epilepsy. These symptoms may be generally described as brief hallucinations, paroxysmal somatic disturbances, automatisms, and dissociative disturbances. The Czech version of LSCL-33 as well as the original English version ${ }^{1}$ shows good psychometric properties and internal consistency (Cronbach's alpha 0.90 with test-retest reliability $r=0.91$ ).

\section{Trauma Symptoms Checklist}

Symptoms of traumatic stress were assessed using the Trauma Symptom Checklist (TSC-40). ${ }^{14}$ TSC-40 is a self-reported questionnaire with 40 items scored on a 4-point Likert scale (total score from 0 to 120). TSC-40 evaluates stress symptoms in adult individuals associated with childhood or adult traumatic experiences and measures aspects of posttraumatic stress and other symptom clusters found in some traumatized individuals. The Czech version of the TSC-40 has high reliability and internal consistency (Cronbach's alpha 0.91 , test-retest reliability after 1 week 0.88 ).

\section{Beck Depression Inventory}

For the assessment of depressive symptoms, Czech version of Beck Depression Inventory (BDI-II) ${ }^{15}$ that represents 21-item questionnaire for assessing depression (Cronbach's alpha 0.89 , test-retest reliability after week 0.85 ) was used. Subjects indicate the degree of their experience of depressive symptoms on a 4-point Likert scale. The scale is sensitive to the changes of the mental state of the individual during the course of time.

\section{Assessment of word-color associations}

The method of word-color associations is based on emotional response to words according to their emotional meaning that may be specifically associated with lighter or darker color and quantified on color scale from 1 to 10 (Figure 1). In the assessment, colors are associated with words that also

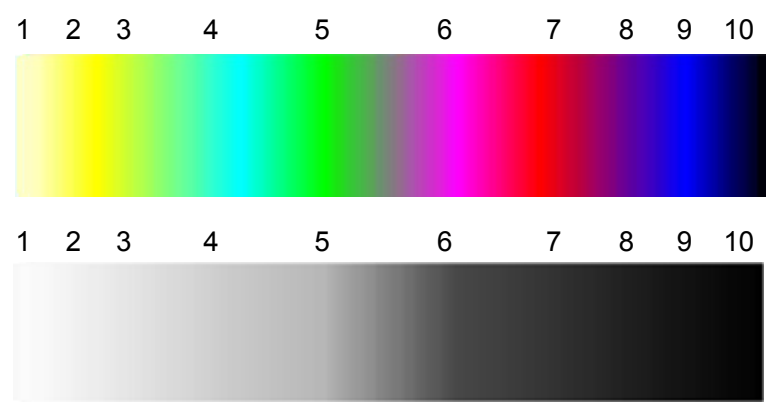

Figure I Color scale used for measurement of color associations in response to word stimuli, which in black-white projection provides gray continuous Likert scale from white to black $(I-I 0)$. 
include a number of critical words, which usually have particular psychological significance that may cause association disturbances. ${ }^{16,17}$ The critical words are designed to recall previous affective associations that modulate new defensive reactions and lead to significant physiological response. ${ }^{16}$ During the experiment, the standard list of 25 stimulus words plus three added words (love, sex, and punishment) were presented in the following order (critical words in italic): 1) brook, 2) lion, 3) book, 4) dark, 5) love, 6) child, 7) table, 8) head, 9) death, 10) boy, 11) illness, 12) hand, 13) mountain, 14) sex, 15) crying, 16) needle, 17) family, 18) cheese, 19) moon, 20) fear, 21) window, 22) street, 23) punishment, 24) salt, 25) man, 26) anger, 27) soldier, and 28) doctor. To each stimulus word three colors in the sequences that they came to mind were associated, and these colors were quantified by three numbers on the color scale. The mean score of these three numbers assigned to the three colors, number of the first associated color (1), and maximum difference (dif) between the two numbers (reflecting maximum and minimum of these 3 numbers associated with the darkest and lightest colors) were used for scoring.

\section{Statistical methods}

Statistical evaluation for the results of word-color associations and other psychometric measures included descriptive statistics and Spearman correlation coefficients. All the methods of statistical evaluation were performed using the software package Statistica version 6.

\section{Results}

The results of descriptive statistics for all included participants indicate a tendency to link the level of darkness on the scale of colors (from white 0 to black 10) with words generally perceived as negative anger (mean $=7.89)$, punishment (mean $=7.68)$, fear $($ mean $=7.65)$, death $($ mean $=7.56)$, disease (mean $=7.09$ ), crying (mean $=6.61$ ) on the other hand the less "dark" (lighter) scores were for example linked to words such as child (mean $=4.04$ ) and family (mean $=4.67$ ).

In addition, the data show specific differences between both subgroups related to occurrences of significant correlations of means, first associated color (1) and the maximum difference of the lightest and darkest associated color (dif) with psychopathological symptoms of temporal lobe epilepsy (LSCL-33), depression (BDI-II), and traumatic stress (TSC-40).

The results in both subgroups indicate specific words correlated with the scores of psychopathological symptoms measured by LSCL-33, BDI-II, and TSC-40. In the first subgroup of participants with high LSCL-33 were significant
Spearman correlations of scores related to associated colors with LSCL-33 (Brook [mean] Spearman $R=0.36, P<0.05$; love (1) $-0.48, P<0.01$; child (1) $-0.42, P<0.01$; cheese [mean] 0.41, $P<0.01$; fear [mean] $-0.39, P<0.05)$ with TSC-40 (table [mean] $-0.43, P<0.05$; table (1) -0.52 , $P<0.01$; death (1) $-0.47, P<0.01$; hand (1) $-0.40, P<0.01$; cheese [dif] 0.45, $P<0.01$; moon [mean] 0.36, $P<0.05$; punishment (1) $-0.43, P<0.05$; doctor (1) $-0.42, P<0.05$ ), and BDI-II (Book [mean] 0.40, $P<0.05$; hand (1) $-0.51, P<0.01$; mountain (1) $-0.46, P<0.05$; moon [mean] 0.45, $P<0.01$; street (1) $0.42, P<0.05$; man (1) $-0.42, P<0.05$ ).

In the second subgroup that included healthy controls with low LSCL-33 score, we found significant correlations for scores associated on the color scale with LSCL-33 (book [mean] -0.34, $P<0.05$; book (1) $-0.44, P<0.01$; child [dif] $-0.45, P<0.01$; sex [dif] 0.33, $P<0.05$; family [mean] $0.36, P<0.05$; moon [mean] $-0.40, P<0.05$; moon [dif] $-0.46, P<0.01$; fear (1) $-0.38, P<0.01$; street [dif] -0.33 , $P<0.05$; punishment (1) $-0.41, P<0.01$ ) with TSC-40 (hand (1) $-0.32, P<0.05$; street (1) $-0.36, P<0.05$; punishment (1) $-0.34, P<0.05$; soldier [mean] $0.37, P<0.05$; doctor (1) $0.40, P<0.05)$. No significant correlations related to scores of associated colors with BDI-II have been found.

It is important to note that we refer to these significant correlations based on single tests, and due to multiple comparisons, the Bonferroni correction should be considered. Nevertheless, the results of the correlations of word-color associations with psychometric measures have nonrandom pattern. In order to avoid the type II errors due to taking assessed correlations as independent we refer to uncorrected statistical significance.

\section{Discussion}

The results of descriptive statistics are congruent with previously reported studies, which suggest that positive emotional meanings are more frequently associated with lighter colors. ${ }^{11,13}$ These findings are in agreement with data that spoken words influence activations in brain visual areas. ${ }^{18}$ The results show specific differences between both subgroups related to occurrences of significant correlations of means, first associated color (1), and the maximum difference of the lightest and darkest associated color (dif) with psychopathological symptoms indicating that the subgroup with higher level of temporal lobe seizure-like symptoms measured by LSCL-33 has higher ability to represent emotional meaning of words by associated colors.

In this context, Terhune et $\mathrm{al}^{6}$ reported that increased excitability in the primary visual cortex using transcranial direct current stimulation applied in five volunteers who 
usually and spontaneously have synesthetic experiences led to a stronger experience of colors connected with words or numbers. Similar findings about increased cortical excitability and synesthesia was also reported by Bolognini et al. ${ }^{19}$ These results are also documented by some case studies, for example, the reported data about Vincent van Gogh, who most likely had synesthetic experiences related to temporal lobe seizures. ${ }^{20}$

Taken together these data support the hypothesis that the associated colors manifest much stronger relationship with LSCL-33 than with the symptoms of traumatic stress and depressive symptoms. This relationship is likely due to seizure-like conditions and increased excitability reflected by the symptoms of limbic irritability (LSCL-33) that may cause increased association connectivity.

\section{Conclusion}

The results indicate specific synesthetic-like mechanism in association processes that reflects psychopathological symptoms related to increased temporo-limbic excitability. Although the results of this study provide promising data for the quantification of projective assessments using word-color associations, further research in large samples with specific age and sex is warranted. This future detailed research could enable to find quantified psychodiagnostic projective assessments of cognitive and affective symptoms related to temporal lobe epilepsy in psychiatric patients. This projective synesthetic-like assessment altogether with LSCL-33 could be helpful for diagnostic consideration of anticonvulsant treatment in patients who do not have abnormalities on scalp electroencephalograms, but might positively respond to antiepileptic medication.

\section{Acknowledgments}

The study was supported by the Charles University grant (PRVOUK and SVV), Project GACR P407/12/1957 and the project "CEITEC - Central European Institute of Technology" (CZ.1.05/1.1.00/02.0068) from the European Regional Development Fund.

\section{Disclosure}

The authors report no conflicts of interest in this work.

\section{References}

1. Teicher MH, Glod CA, Surrey J, Swett C Jr. Early childhood abuse and limbic system ratings in adult psychiatric outpatients. J Neuropsychiatry Clin Neurosci. 1993;5:301-306.

2. Teicher MH, Andersen SL, Polcari A, et al. The neurobiological consequences of early stress and childhood maltreatment. Neurosci Biobehav Rev. 2003;27:33-44.

3. Teicher MH, Tomoda A, Andersen SE. Neurobiological consequences of early stress and childhood maltreatment: are results from human and animal studies comparable? Ann N Y Acad Sci. 2006;1071:313-323.

4. Roberts RJ, Gorman LL, Lee GP, et al. The phenomenology of multiple partial seizure like symptoms without stereotyped spells: an epilepsy spectrum disorder? Epilepsy Res. 1992;13:167-177.

5. Ramachandran VS, Hubbard EM. Synaesthesia: a window into perception, thought and language. J Conscious Stud. 2001;8:3-34.

6. Terhune DB, Tai S, Cowey A, Popescu T, Cohen Kadosh R. Enhanced cortical excitability in grapheme-color synesthesia and its modulation. Curr Biol. 2011;21(23):2006-2009.

7. Cytowic RE. Synesthesia: A Union of the Senses. 2nd ed. Cambridge: MIT Press; 2002.

8. Martino G, Marks LE. Synesthesia: strong and weak. Psychol Sci. 2001;10:61-65.

9. Simner J. Why are there different types of synesthete? Front Psychol. 2013;4:558.

10. Marks LE. The Unity of the Senses: Interrelations Among the Modalities. New York: Academic Press; 1978.

11. Cohen Kadosh R, Sagiv N, Linden DE, Robertson LC, Elinger G, Henik A. When blue is larger than red: colors influence numerical cognition in synesthesia. J Cogn Neurosci. 2005;17(11):1766-1773.

12. Kuhbandner C, Pekrun R. Joint effects of emotion and color on memory. Emotion. 2013;13(3):375-379.

13. Okubo M, Ishikawa K. Automatic semantic association between emotional valence and brightness in the right hemisphere. Cogn Emot. 2011;25(7):1273-1280.

14. Briere J. Psychometric review of the Trauma Symptom Checklist-40. In: Stamm BH, editor. Measurement of Stress, Trauma, and Adaptation. Lutherville: Sidran Press; 1996.

15. Beck AT, Brown G, Steer RA. Beck Depression Inventory II Manual. San Antonio, TX: The Psychological Corporation; 1996.

16. Jung CG. The association method. Am J Psychol. 1910;31:219-269.

17. Kondas O. Associative Experiment. Bratislava: Psychodiagnostika; 1989.

18. Nunn JA, Gregory LJ, Brammer M, et al. Functional magnetic resonance imaging of synesthesia: activation of V4/V8 by spoken words. Nat Neurosci. 2002;5(4):371-375.

19. Bolognini N, Miniussi C, Gallo S, Vallar G. Induction of mirror-touch synaesthesia by increasing somatosensory cortical excitability. Curr Biol. 2013;23(10):R436-R437.

20. Voskuil PH. Van Gogh's disease in the light of his correspondence. Front Neurol Neurosci. 2013;31:116-125.
Neuropsychiatric Disease and Treatment

\section{Publish your work in this journal}

Neuropsychiatric Disease and Treatment is an international, peerreviewed journal of clinical therapeutics and pharmacology focusing on concise rapid reporting of clinical or pre-clinical studies on a range of neuropsychiatric and neurological disorders. This journal is indexed on PubMed Central, the 'PsycINFO' database and CAS,

\section{Dovepress}

and is the official journal of The International Neuropsychiatric Association (INA). The manuscript management system is completely online and includes a very quick and fair peer-review system, which is all easy to use. Visit http://www.dovepress.com/testimonials.php to read real quotes from published authors. 